\title{
Supporting Information for: Data-driven discovery and synthesis of high entropy alloy hydrides with targeted thermodynamic stability
}

\author{
Matthew Witman*1, Gustav Ek ${ }^{2}$, Sanliang Ling ${ }^{3}$, Jeffery Chames ${ }^{1}$, Sapan Agarwal ${ }^{1}$, \\ Justin Wong ${ }^{1}$, Mark D. Allendorf ${ }^{1}$, Martin Sahlberg*2 ${ }^{* 2}$ and Vitalie Stavila*1 \\ ${ }^{1}$ Sandia National Laboratories, Livermore, California 94551, USA \\ ${ }^{2}$ Department of Chemistry - Ångström Laboratory, Uppsala University, Box 523, \\ SE-75120, Uppsala, Sweden \\ ${ }^{3}$ Advanced Materials Research Group, Faculty of Engineering, University of \\ Nottingham, University Park, Nottingham, NG7 2RD, UK
}

\section{S1. Thermodynamic model details}

This section provides extra details on the training and performance of the hydride thermodynamic ML models. Model training and results can all be reproduced via the link to the Jupyter notebook in Section S4.

\section{S1.1. Data cleaning}

The cleaned, ML-ready version of the HydPARK dataset was used as training data for the thermodynamics models in this work. Details on the data cleaning can be found in Ref. 1.

\section{S1.2. Model training and performance}

Following the workflow described in Ref. 1, we train gradient boosting tree (GBT) regressors as implemented in scikit-learn ${ }^{2}$ to predict $\Delta H, \Delta S$, and $\ln \left(P_{e q}^{o} / P_{o}\right)$. The hyperparameters for the GBT regressors are provided in our open source Jupyter notebooks (Section S4). Two significant improvements upon the original workflow were made, as detailed in the manuscript. These included augmenting the HydPARK database with thermodynamic data on TiVZrNbHf and TiVZrNb to create HydPARK +, and augmenting Magpie features with quantities that reflect the stability of a composition's constituent binary hydrides, i.e. $\Delta \bar{H}_{b h}$. The performance of these models is summarized in Figure S1. For the concatenated results of 10 -fold validation, the training and validation parity plots are shown in columns one and two for all three thermodynamic models. The third column shows the relative feature importance averaged across all 10-fold models. Column four highlights how the validation error depends on the target quantity distribution.

The validation MAE averaged across all 10 -folds are $1.44,5.5 \mathrm{~kJ} / \mathrm{mol} \cdot \mathrm{H}_{2}$, and $13.7 \mathrm{~J} /\left(\mathrm{mol} \cdot \mathrm{H}_{2} \mathrm{~K}\right)$ for $\ln \left(P_{e q}^{o} / P_{o}\right), \Delta H$, and $\Delta S$, respectively, which are $4 \%, 10 \%$, and $2 \%$ lower than those reported in our original work. While modest, this indicates that data and feature augmentation successfully

*mwitman@sandia.gov ; martin.sahlberg@kemi.uu.se ; vnstavi@sandia.gov 

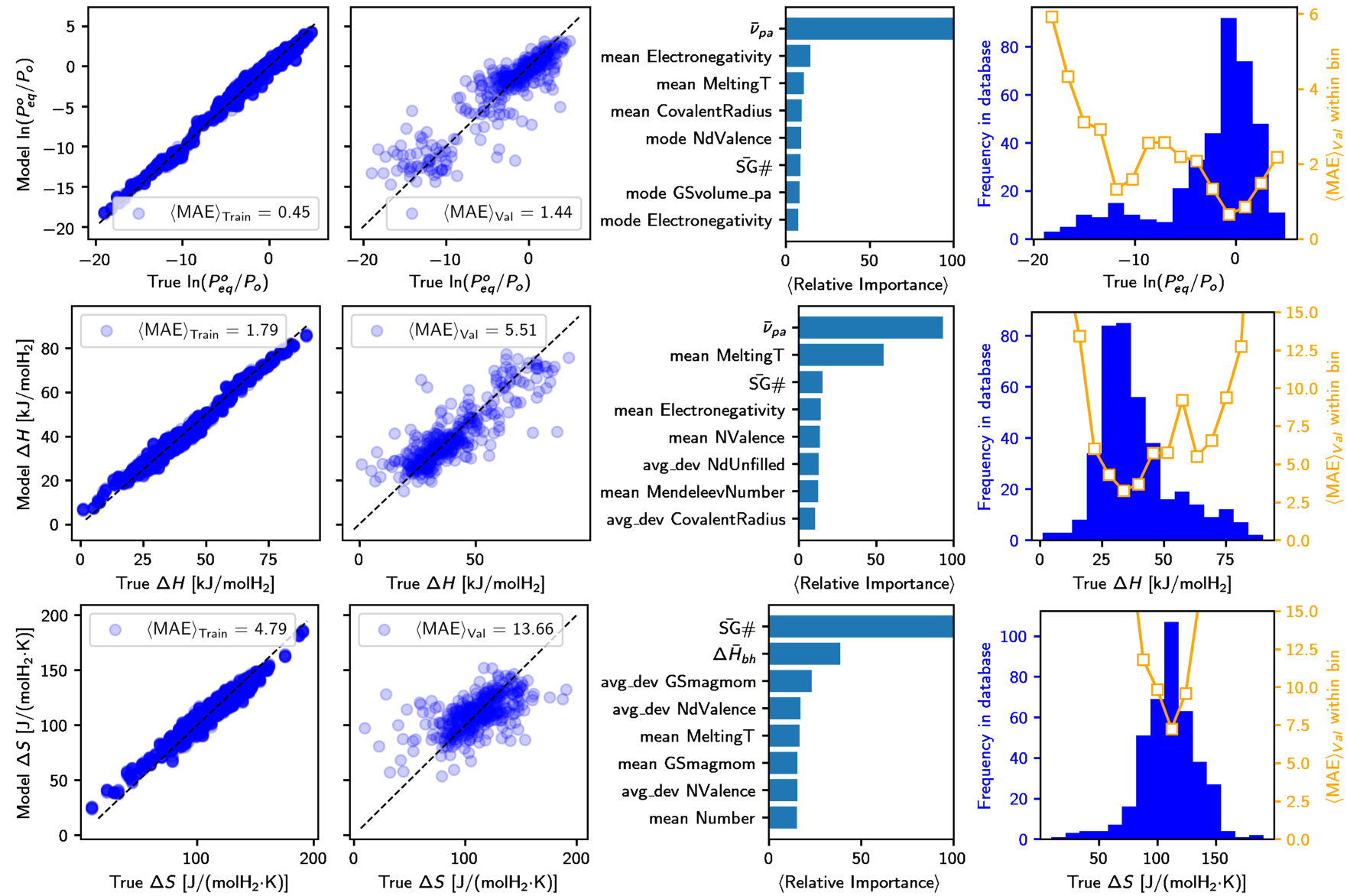

Figure S1: The training parity plot (column 1), validation parity plot (column 2), averaged feature importance (column 3), and the validation error across the target distribution (column 4) for the concatenated results of a 10 -fold validation experiment. $\ln \left(P_{e q}^{o} / P_{o}\right), \Delta H$, and $\Delta S$ models correspond to rows 1,2 , and 3 , respectively. 

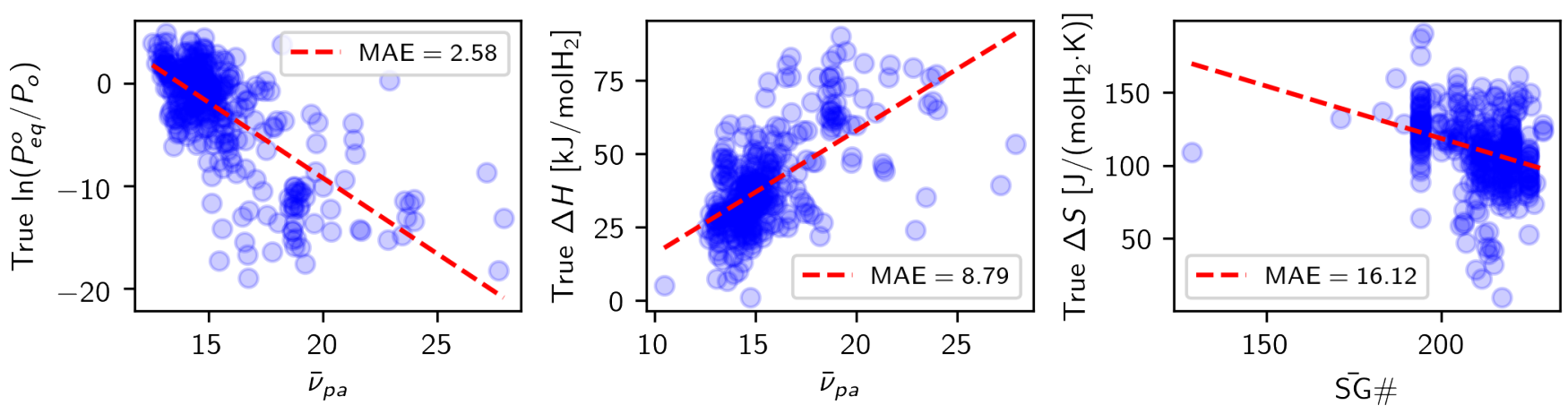

Figure S2: Thermodynamic target properties $\ln \left(P_{e q}^{o} / P_{o}\right), \Delta H$, and $\Delta S$ plotted vs. their most important variable identified by the GBT models. Red dashed lines show a linear fit and the corresponding MAE.

improve model fidelity and hints that continued augmentation of the HydPARK database with both current and future HEA hydrides will provide even greater model improvement. The second most important feature in the $\Delta S$ model is $\Delta \bar{H}_{b h}$, which indicates how carefully hand crafting additional physically meaningful features helps generate better compositional ML models.

Due to the dominant importance of $\bar{\nu}_{p a}$ in the $\ln \left(P_{e q}^{o} / P_{o}\right)$ and $\Delta H$ models, it is worthwhile to compare the predictive capabilities of the GBT models vs. a simple linear model for each thermodynamic property that uses only its most important feature from the GBT model. Performing a linear regression of all three target properties on these variables is shown in Figure S2, which results in substantial increases in MAE for these simpler models. Clearly these primary correlations cannot serve as replacement for the more accurate GBT models; nonetheless they still provide first order design rules that can be easily interpreted.

\section{S1.3. HydPARK and rHEA distribution mismatch}

Adding the thermodynamic measurements from Ref. 3 for TiVZrNbHf $\left(\bar{\nu}_{p a}=18.7\right)$ and TiVZrNb $\left(\bar{\nu}_{p a}=17.8\right)$ to the original HydPARK training data helps address the distributional mismatch of $\bar{\nu}_{p a}$ with the rHEA data, as illustrated in Figure S3. Adding additional examples in this low $\bar{\nu}_{p a}$ training data regime provides a heuristic argument why training a model on HydPARK vs. HydPARK+ systematically affects thermodynamic predictions (Figure 1b). Due its strong, log-linear correlation with hydride stability, this $\bar{\nu}_{p a}$ distributional mismatch would also suggest a distribution mismatch between HydPARK thermodynamic data and ML predictions on rHEA materials. Indeed, Figure S4 shows the distribution of rHEA materials thermodynamic target quantities (blue), which differs significantly from the predicted rHEA thermodynamic quantities (for both models trained either on HydPARK or HydPARK+). This exercise justifies the need to continually acquire high quality thermodynamic data on metal hydrides to improve these thermodynamic models and facilitate novel material discovery exercises with greater efficiency.

\section{S2. Phase prediction model details}

This section provides additional training and performance details on the GBT and Alternative Weights Explainable-ML (AWE-ML) classifiers that were trained for the phase prediction task presented in the manuscript. Model training and results can all be reproduced via the link to the Jupyter notebook in Section S4. 


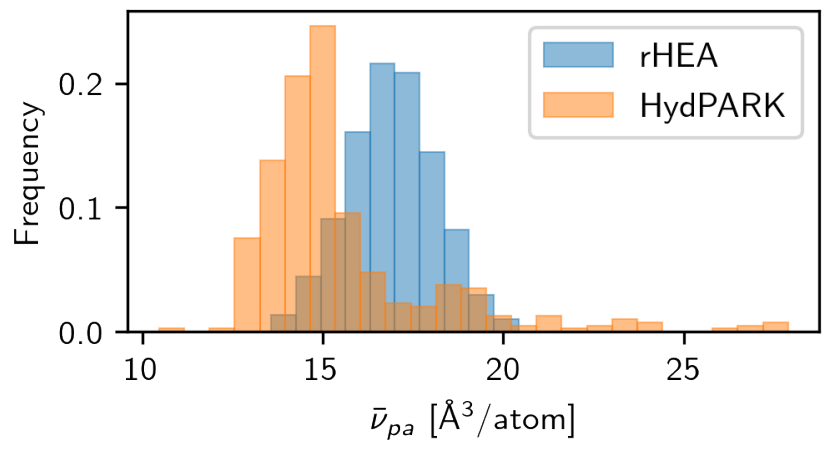

Figure S3: Distribution of $\bar{\nu}_{p a}$ values in the HydPARK training data and the new rHEA dataset.
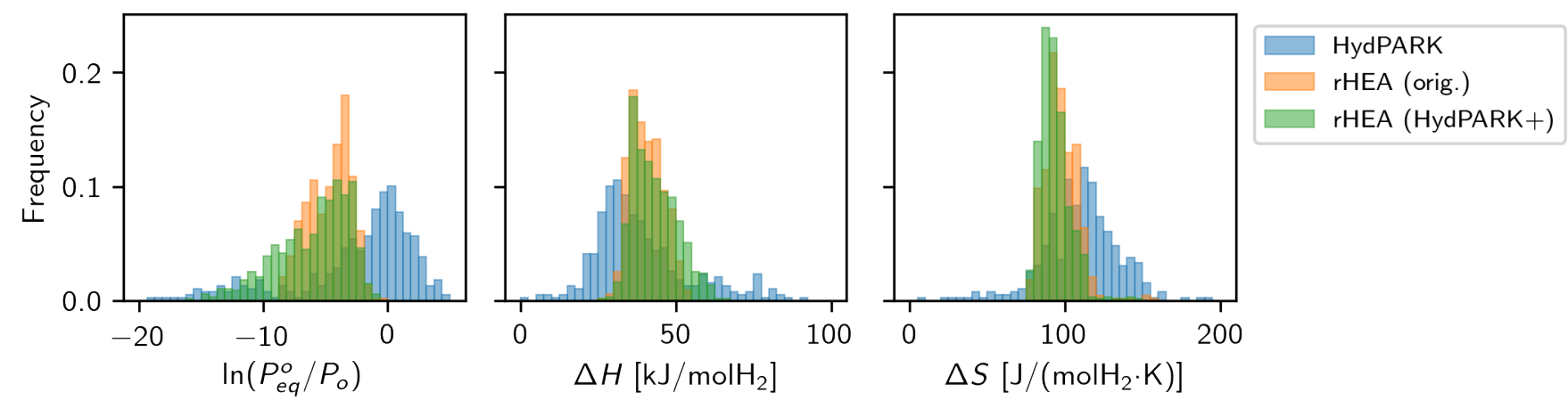

Figure S4: The distribution of HydPARK target thermodynamic quantities versus the distribution of predicted rHEA thermodynamic quantities for models trained on both HydPARK and HydPARK+ datasets.

\section{S2.1. Data cleaning}

The HEA dataset of Miracle et al. reports 400 HEA compositions and their assigned target class of 'IM', 'SS', or 'SS+IM', which refers to the experimentally observed intermetallic (IM), single-phase solid solution (SS), or impure mixture of SS+IM, respectively. Additional information is included in the dataset, such as synthesis conditions for some entries and the crystal packing type for SS entries (i.e., FCC or BCC). This introduces additional complexities; for example, there are several examples of identical compositions with different target classes due to differing synthesis conditions. Accounting for this is outside the scope of the current work. We are mainly focused on whether a single-phase SS can be achieved (regardless of synthesis conditions), and so we rank the target classes from most important to least: 'SS', 'SS+IM', and 'IM'. For duplicate compositions with differing target classes, the label kept for the training data is the most important. So, if composition X appears twice in the dataset with target class 'SS' in one entry and 'SS+IM' in the other, the label kept for the training data is 'SS'. Similar to the thermodynamic models, each HEA composition is featurized with Magpie.

\section{S2.2. $\quad$ GBT model training, performance, and feature importance}

The Hyperopt package ${ }^{4}$ was used to optimize the hyperparameters of the GBT model, which achieved an average accuracy of $74 \%$ across a 5 -fold validation. Figure S5 shows both the train/validation curves and the relative feature importance for each $\mathrm{k}$-fold model under the optimal hyperparameter 

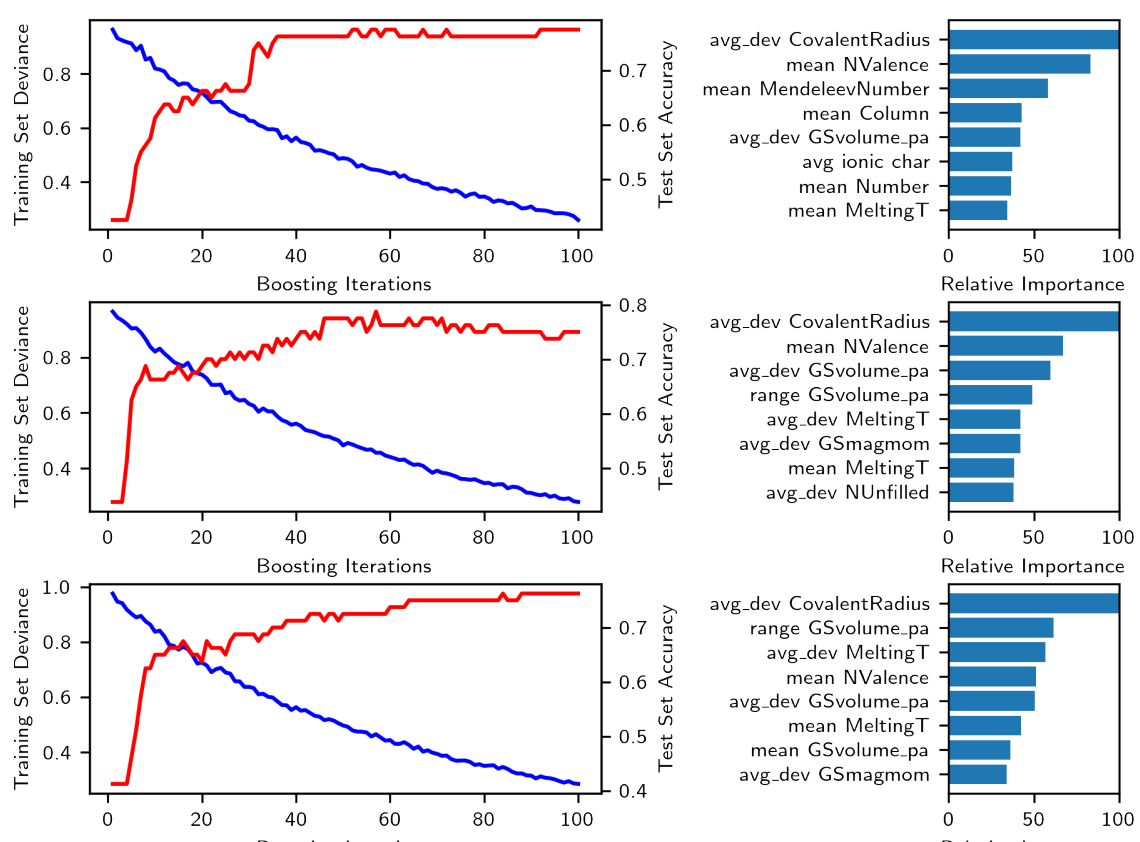

avg_dev CovalentRadius range GSvolume_pa avg_dev MeltingT mean NValence avg_dev GSvolume_pa mean MeltingT
mean GSvolume_pa avg_dev GSmagmom
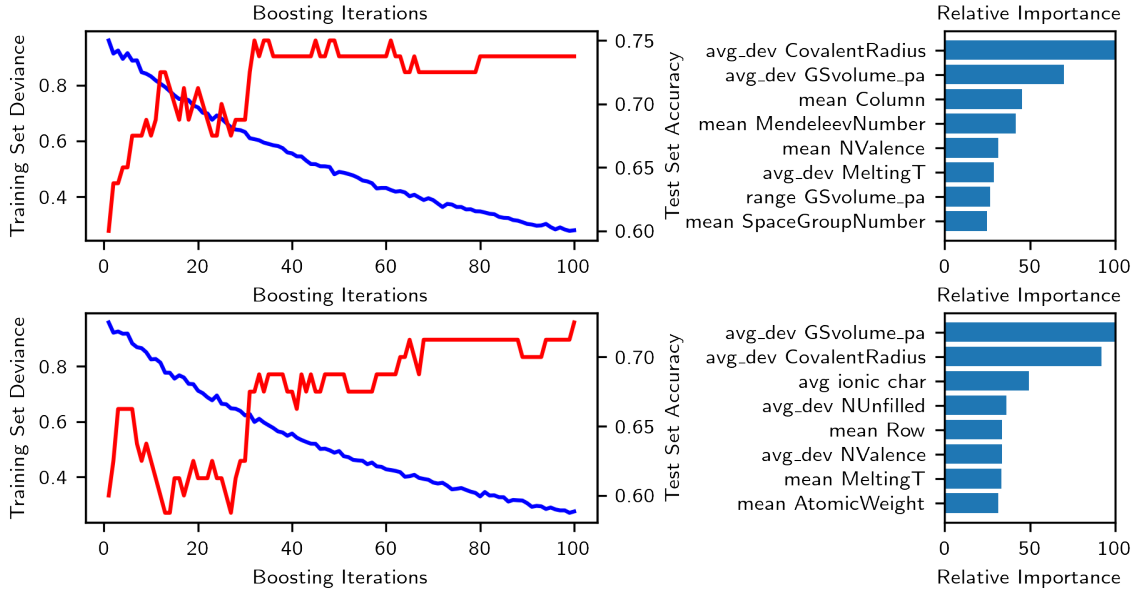

avg_dev GSvolume_pa avg_dev CovalentRadius avg ionic char avg_dev NUnfilled mean Row avg_dev NValence
mean MeltingT mean AtomicWeight Relative Importance 'SS+IM', or 'IM' based on the Miracle et al. training dataset. The feature importance of each model shows that GBT recovers long-standing physical relationships like Hume-Rothery rules.

set. As has already been established by Hume-Rothery $\operatorname{rules}^{5}$ and other recent analyses ${ }^{6}$ an important criteria for determining SS forming ability is the difference in atomic sizes of elements in a given composition (denoted by "avg_dev CovalentRadius" $=\sum f_{i}\left|R_{i}-\sum f_{i} R_{i}\right|$, where $f_{i}$ is the composition fraction of element $i$ and $R_{i}$ is the covalent radius). This indicates that this approach recovers previously known physical design rules, and, as HEA databases become more comprehensive, more advanced interpretable ML analyses may help improve such design rules.

\section{S3. DFT settings}

All density functional theory (DFT) calculations, including structural optimizations and total energy calculations, were performed using the Vienna Ab initio Simulation Package. ${ }^{7}$ We used a plane-wave 
basis set with a kinetic energy cutoff of $400 \mathrm{eV}$ to expand the wave functions, and the PerdewBurke-Ernzerhof functional ${ }^{8}$ with the projector augmented wave method ${ }^{9,10}$ were used to solve the Kohn-Sham equations. A first-order Methfessel-Paxton smearing ${ }^{11}$ with a width of $0.2 \mathrm{eV}$ was used to improve the convergence of electronic self-consistent field calculations. All DFT calculations have been performed using a k-point mesh with a spacing of ca. $0.20 \AA^{-1}$. An energy convergence threshold of $10^{-4} \mathrm{eV}$ was used for all total energy calculations. Due to the enormous number of structures that need to be considered, a relatively loose structural optimization convergence criterion was used, where the structural optimizations, including cell parameters and atomic positions, were considered converged if all interatomic forces fall below $0.05 \mathrm{eV} / \AA$. The structures which we have considered include HEAs with different chemical compositions, and for each HEA composition, a large number of metal alloy configurations with different metal distributions (in both FCC and BCC structures) were considered, and for each selected metal alloy configuration, a large number of hydride configurations with different hydrogen loading and distribution were considered. All the DFT calculations were performed in sufficiently large supercells to account for the statistically

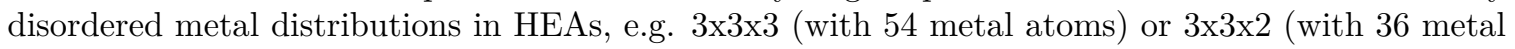
atoms) supercells were used for BCC structures and $3 \times 2 \times 2$ supercells (with 48 metal atoms) were used for FCC structures. The starting HEA alloy and hydride configurations were generated using our HEAhydrideTK code (Section S4).

\section{S4. Code Availability}

The code necessary to reproduce the metal hydride thermodynamic ML models and visualize their results is provided at https://github.com/mwitman1/HEAhydrideML.

The code necessary to reproduce the phase prediction ML model and visualize its results is provided at https://github.com/mwitman1/HEAphaseML.

A final summary of the predicted rHEA properties (both hydriding thermodynamics and phase prediction) is provided in rHEA_final_predictions.csv in the supporting files.

The code necessary to generate the starting HEA alloy and hydride configurations for DFT relaxation is provided at https://github.com/mwitman1/HEAhydrideTK.

\section{S5. Experimental Methods}

\section{S5.1. HEA synthesis}

The alloys were synthesized by melting lumps of $\mathrm{Ti}$ (Goodfellow, $99.99 \%$ metals basis), V (ChemPur, 99.9\% metals basis), Nb (Alfa Aesar, 99.95\% metals basis), Zr (Chempur, 99.8\% metals basis, excluding Hf), Hf (Chempur, 99.8\% metals basis, maximum 4\% Zr), Ta (Goodfellow, 99.9\% metals basis), Cr (Alfa Aesar, 99.995\% metals basis), Mo (Goodfellow, 99.9\% metals basis), Al (Gränges, 99.999\% metals basis), $\mathrm{Pd}$ (Hereaus, $99.9 \%$ metals basis) in an electric arc furnace in Ar atmosphere. Elements with low vapor pressure $(\mathrm{Al}, \mathrm{Cr})$ were pre-alloyed before final synthesis to avoid evaporation. To ensure chemical homogeneity, the samples were remelted five times and flipped between each melting. The mass losses were in all cases less than $0.1 \mathrm{wt} \%$ and thus the resultant composition can be considered very close to the nominal. Some of the ingots were then filed to powder with a metal file for X-ray diffraction analysis and the rest cut into smaller pieces for hydrogenation. 


\section{S5.2. Alloy characterization}

\section{S5.2.1. X-ray diffraction (XRD)}

Laboratory X-ray diffraction was carried out on a Bruker D8 advance with $\mathrm{Cu} \mathrm{K} \alpha$ radiation equipped with a LynxEye XE-T detector operating in Bragg-Brentano geometry, with the powders placed on zero-background Si-wafer sample holders. The data was analyzed by the Rietveld method implemented in the software Topas v6 Academic. ${ }^{12}$ Peak shapes were modelled by Thompson-CoxHastings pseudo Voigt function ${ }^{13}$ and the background by a six-order Chebyshev polynomial. Site occupancies were fixed to the nominal composition in all cases.

\section{S5.2.2. Energy Dispersive X-Ray Spectroscopy (EDS)}

A JEOL JSM-7600F Thermal Field Emission Electron Microscope was used to obtain SEM images, and an Oxford X-Max 80 detector with Aztec Software used to obtain EDS maps. The conditions use for both the SEM images and the EDS maps were: $\mathrm{HT}=15 \mathrm{kV}, \mathrm{WD}($ nominal $)=8 \mathrm{~mm}$, Probe setting $=6$. The sample was deposited onto carbon tape on an Aluminum stub, and no coating was applied. Areas chosen for analysis were fairly level and flat for best imaging results. This also pertains to obtaining the best EDS detection (count rate), as dramatic topography often results in shadowing that may be interpreted as an area depleted in an element. For AlTiVCr and AlTiVNbTa, the SEM images in Figure S6 and Figure S7, respectively, show the location of the EDS maps and the complete spectrum for elemental composition analysis. The EDS maps for AlTiVCr are shown in Figure S8, while those for AlTiVNbTa were shown in Figure 3. 
(a) Alloy
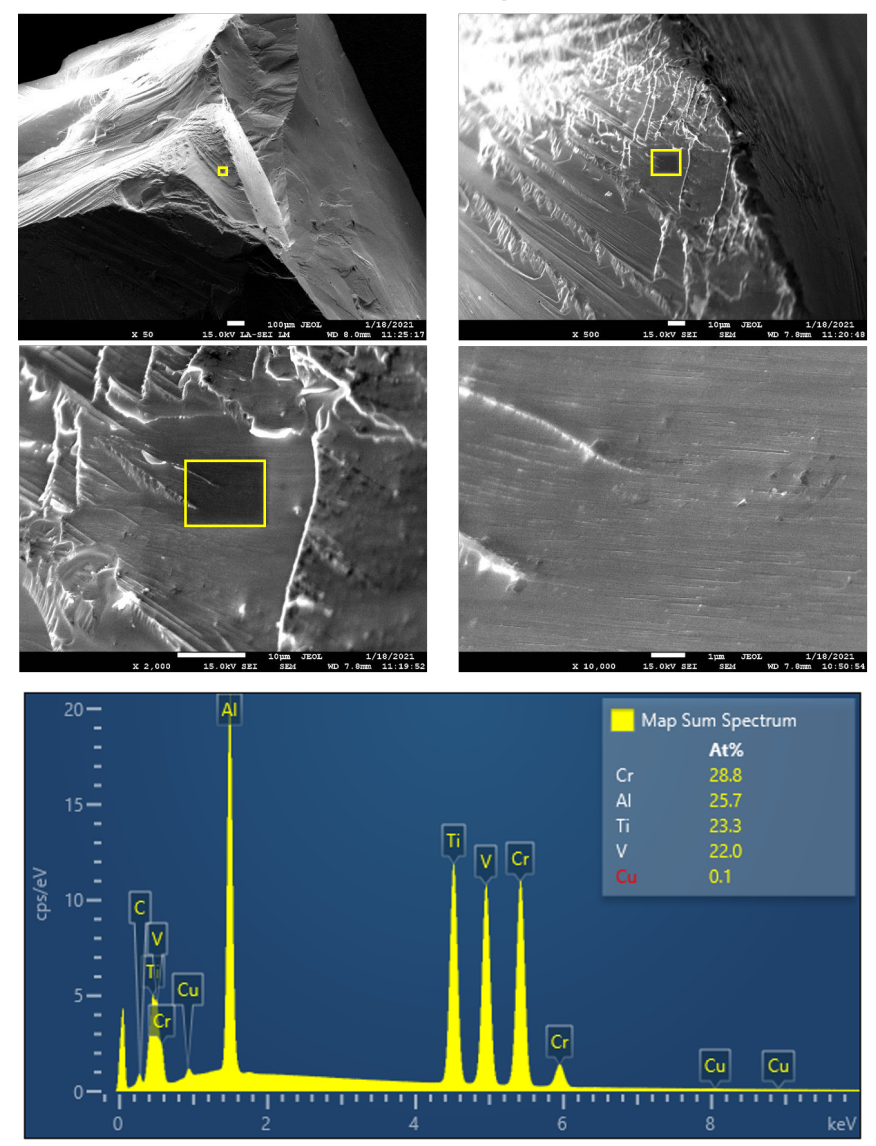

(b) Post- $\mathrm{H}_{2}$
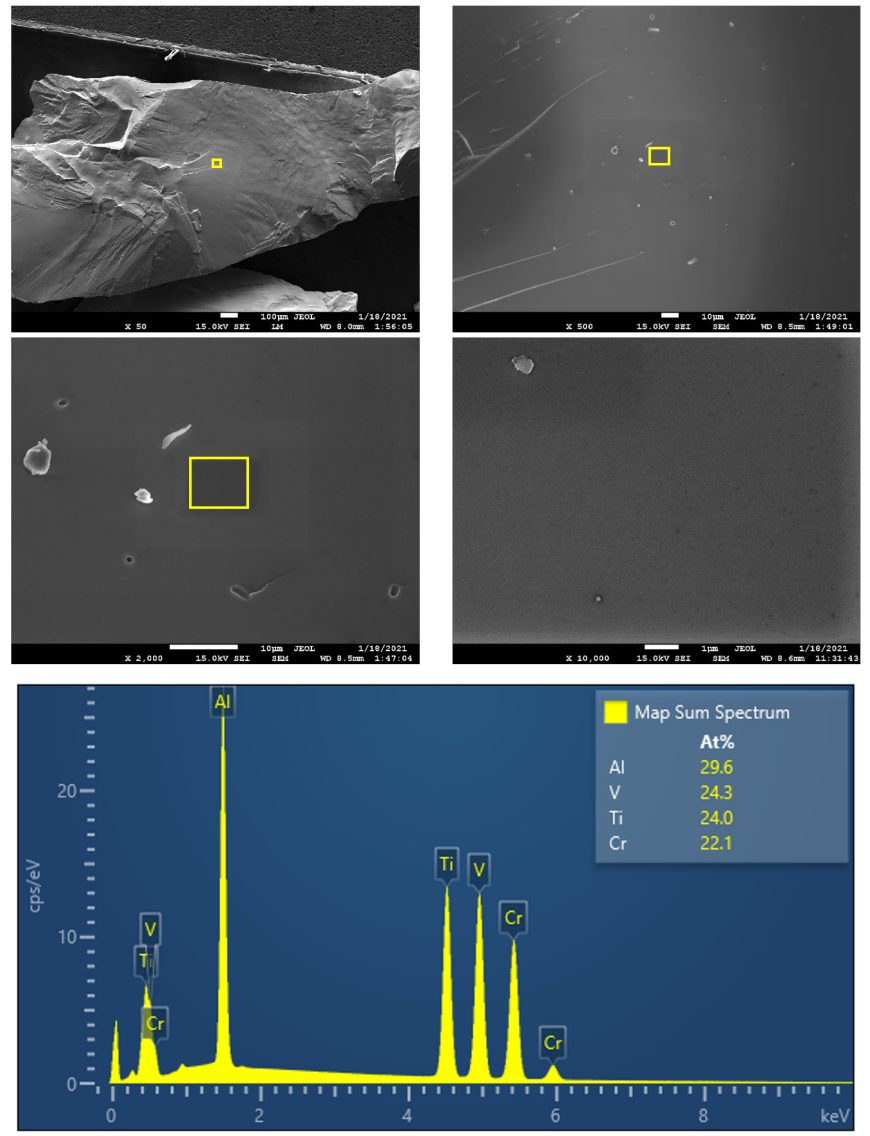

Figure S6: 10000x EDS map location and complete spectrum for AlTiVCr for (a) the as synthesized alloy and (b) the post-hydrided sample. 
(a) Alloy

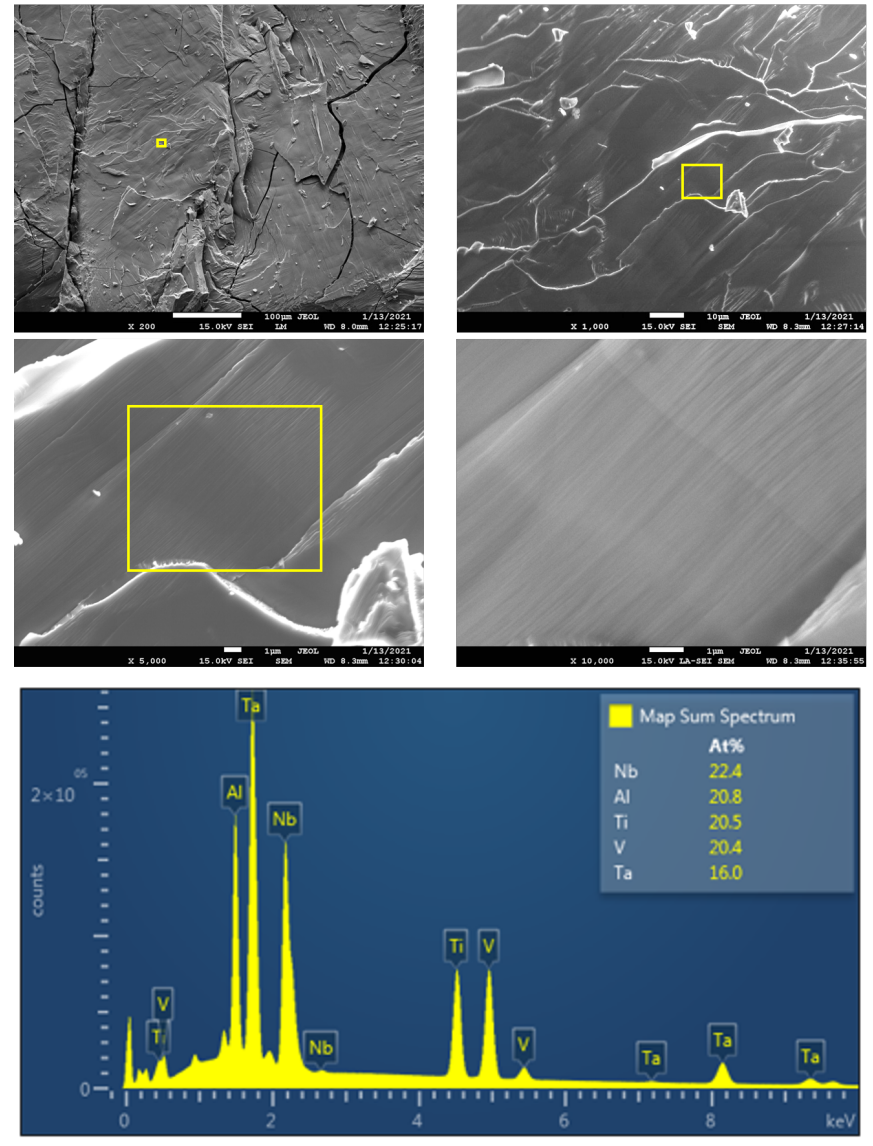

(b) Post- $\mathrm{H}_{2}$
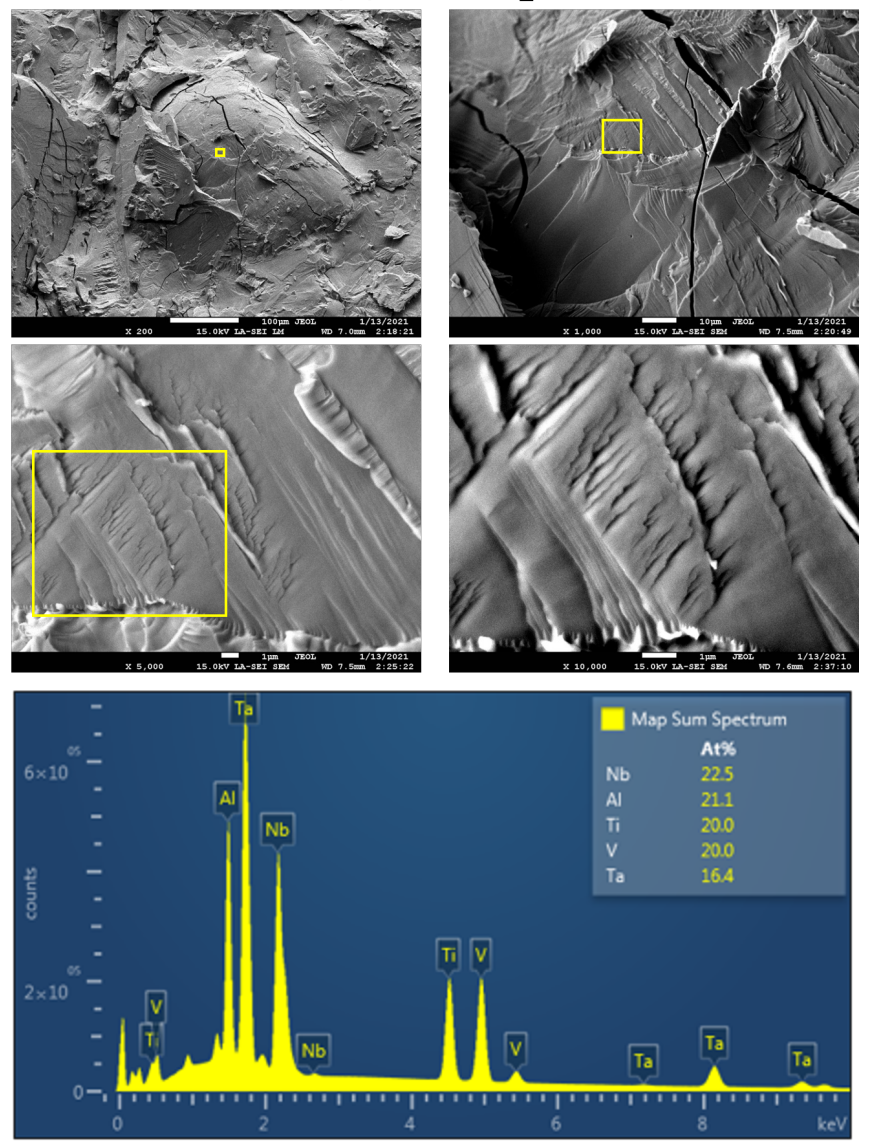

Figure S7: 10000x EDS map location and complete spectrum for AlTiVNbTa for (a) the as synthesized alloy and (b) the post-hydrided sample. 

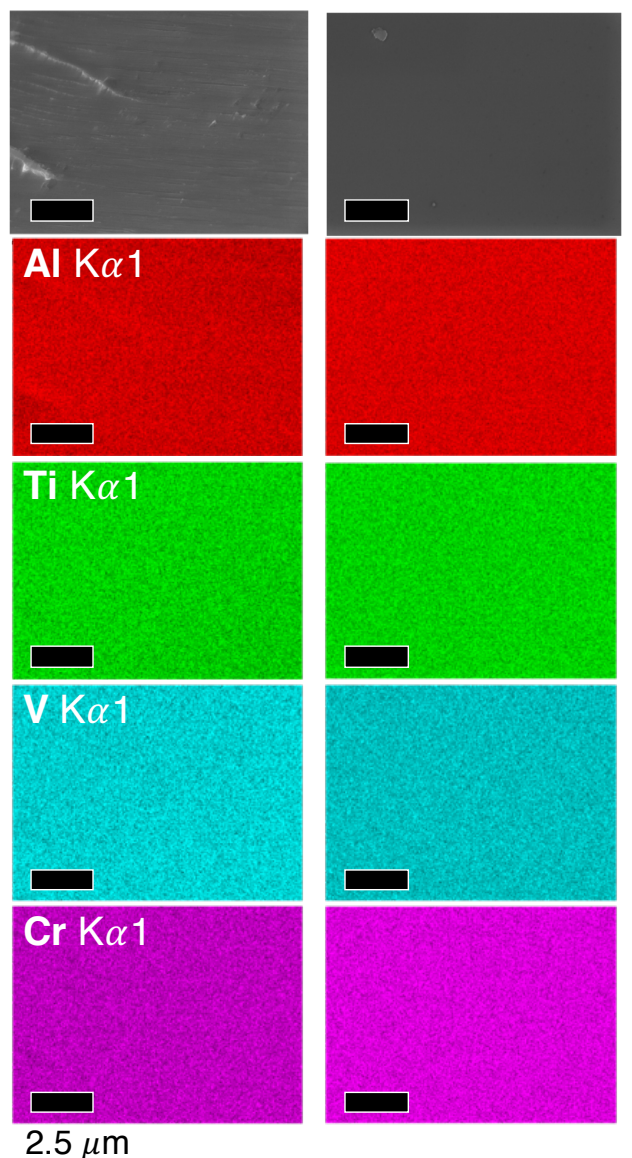

Figure S8: 10000x EDS maps for AlTiVCr for the as synthesized alloy (left column) and the post-hydrided sample (right column). 


\section{S5.3. HEA hydride thermodynamic measurements}

\section{S5.3.1. PCT data collection}

Pressure composition-temperature (PCT) isotherms were collected using a Setaram PCTPro instrument. Samples were loaded into high-pressure vessels, sealed with stainless-steel gaskets, and put under vacuum before thermal activation at $753 \mathrm{~K}$. The thermocouple measuring the temperature is located outside of the stainless-steel pressure vessel in a thermocouple well to minimize the thermal gradients between the samples and the vessel exterior. The pressure transducers had an accuracy of $\pm 1 \%$. The PCT experiments were conducted isothermally between 562 to $609 \mathrm{~K}$ to ensure that the kinetics of hydrogen uptake are fast enough to reach chemical equilibrium.

\section{S5.3.2. van't Hoff analysis}

The enthalpy and entropy of hydrogenation were determined using a published hybrid PCT/van't Hoff method by determining the equilibrium pressure $P_{1}$ of a sample at a temperature $T_{1}$ by measuring a full isotherm, then heating the sample to temperature $T_{2}$, pressurizing the sample with hydrogen to a pressure calculated value and letting the system come to chemical equilibrium in time at a constant temperature to determine equilibrium pressure $P_{2}{ }^{3}$ The equilibrium hydrogen pressure $P_{e q}$ at a given temperature $T$ is determined by the van't Hoff equation

$$
\ln \left(P_{e q} / P_{o}\right)=-\frac{\Delta H}{R T}+\frac{\Delta S}{R},
$$

where $P_{o}$ and $R$ represent the standard atmospheric pressure and the universal gas constant, respectively.

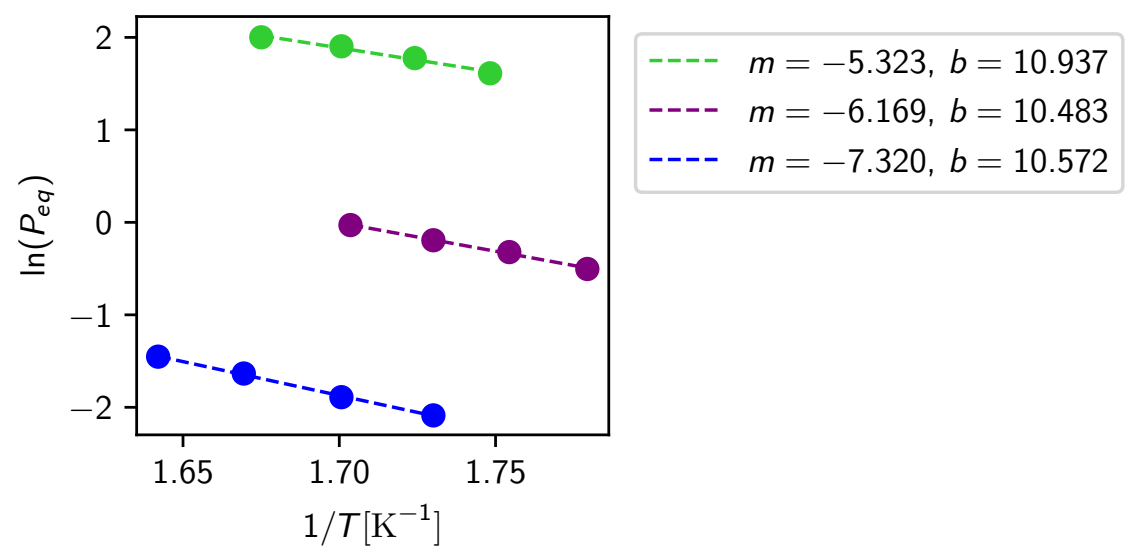

Figure S9: van't Hoff plots used to determine $\Delta H$ and $\Delta S$ AlTiVCr (green) AlTiVNbTa (purple), and TiVZrNbHf (blue). Slopes $(m)$ and intercepts $(b)$ of the linear fits are shown in the legend.

\section{References}

[1] M. Witman, S. Ling, D. M. Grant, G. S. Walker, S. Agarwal, V. Stavila, and M. D. Allendorf, "Extracting an Empirical Intermetallic Hydride Design Principle from Limited Data via Interpretable Machine Learning," J. Phys. Chem. Lett., vol. 11, pp. 40-47, jan 2020.

[2] F. Pedregosa, G. Varoquaux, A. Gramfort, V. Michel, B. Thirion, O. Grisel, M. Blondel, P. Prettenhofer, R. Weiss, V. Dubourg, J. Vanderplas, A. Passos, D. Cournapeau, M. Brucher, 
M. Perrot, and E. Duchesnay, "Scikit-learn: Machine learning in Python," Journal of Machine Learning Research, vol. 12, pp. 2825-2830, 2011.

[3] G. Ek, M. M. Nygård, A. F. Pavan, J. Montero, P. F. Henry, M. H. Sørby, M. Witman, V. Stavila, C. Zlotea, B. C. Hauback, and M. Sahlberg, "Elucidating the Effects of the Composition on Hydrogen Sorption in TiVZrNbHf-Based High-Entropy Alloys," Inorg. Chem., vol. 60, pp. 1124-1132, jan 2021.

[4] J. Bergstra, D. Yamins, and D. D. Cox, "Making a science of model search: Hyperparameter optimization in hundreds of dimensions for vision architectures," in 30th Int. Conf. Mach. Learn. ICML 2013, no. PART 1, pp. 115-123, International Machine Learning Society (IMLS), 2013.

[5] W. Hume-Rothery, "The Structure of Metals and Alloys," Nature, vol. 138, pp. 7-8, jul 1936.

[6] S. Guo, Q. Hu, C. Ng, and C. Liu, "More than entropy in high-entropy alloys: Forming solid solutions or amorphous phase," Intermetallics, vol. 41, pp. 96-103, oct 2013.

[7] G. Kresse and J. Furthmüller, "Efficient iterative schemes for ab initio total-energy calculations using a plane-wave basis set," Phys. Rev. B, vol. 54, pp. 11169-11186, Oct. 1996.

[8] J. P. Perdew, K. Burke, and M. Ernzerhof, "Generalized gradient approximation made simple," Phys. Rev. Lett., vol. 77, pp. 3865-3868, Oct. 1996.

[9] P. E. Blöchl, "Projector augmented-wave method," Phys. Rev. B, vol. 50, pp. 17953-17979, Dec. 1994.

[10] D. Hobbs, G. Kresse, and J. Hafner, "Fully unconstrained noncollinear magnetism within the projector augmented-wave method," Phys. Rev. B, vol. 62, pp. 11556-11570, Nov. 2000.

[11] M. Methfessel and A. T. Paxton, "High-precision sampling for brillouin-zone integration in metals," Phys. Rev. B, vol. 40, pp. 3616-3621, Aug. 1989.

[12] A. A. Coelho, "TOPAS and TOPAS-Academic : an optimization program integrating computer algebra and crystallographic objects written in C++," J. Appl. Crystallogr., vol. 51, pp. 210218 , feb 2018.

[13] P. Thompson, D. E. Cox, and J. B. Hastings, "Rietveld refinement of DebyeScherrer synchrotron X-ray data from $\mathrm{Al}_{2} \mathrm{O}_{3}$, , J. Appl. Crystallogr., vol. 20, pp. 79-83, apr 1987. 